\title{
Research on Socket Communication System Based on Zigbee Wireless Sensor Network
}

\author{
Yujia Wang \\ School of Electrical and Information Engineering;Beijing University of Civil Engineering and \\ Architecture \\ Beijing, 100044, China \\ E-mail: wangyujialstu.bucea.edu.cn
}

\section{Jia Wang}

School of Electrical and Information Engineering;Beijing University of Civil Engineering and Architecture

Beijing, 100044, China

The data of temperature and humidity inside and outside the building is important forbuilding energy consumption analysis. Obtaining, storing and monitoring these data in a timely, accurate and efficient way is of great significance for reducing energy consumption of buildings and achieving sustainable development. The temperature and humidity data collected through the Wireless Sensor Network(WSNs) based on the ZigBee protocol is stored in the SQLCE embedded database of the base station.A remote user is connected to the base station to obtain data., To realize the real-time communication, Internet communication is the most efficient way. Therefore we developed a wireless sensor network remote monitoring system based on Socket network programming. The research group developed a wireless sensor network system as the hardware platform under theVisual Studio 2005 development environment. They combined the technology development of embedded database SQLCE with Sockets socket. C\# programming language is utilized to achieve real-time display of wireless sensor network data remote computer storage and query functions. This research has important practical significance for real-time data sharing based on wireless sensor network technology. Furthermore, it can reduce building energy consumption and broaden the application areas of wireless sensor networks as well. 


\section{Introduction}

In recent years, wireless sensor network (wireless sensor networks, WSNs) has become the frontier and hot research direction to the world. A large number of wireless sensor networks have been put into commercial use.Current research on the wireless sensor network focuses on the network architecture, network communication protocol, network security management and so on. The research of sensor information visualization is relatively small and mostly established on the specific application environment with poor versatility and portability[1]. It can not be directly applied on other platforms.

In accordance with the current research status and requirements of wireless sensor networks, research group developed a remote monitoring system for building information in wireless sensor networks based on Socket network programming.Using the wireless sensor network with the air quality parameters of the system and gathering in an air conditioning room, remote clients can obtain real-time and fast data collected in Internet and other indoor environments just with a computer which can be connected to the network. This method can realize wireless sensor network data acquisition and remote sharing, which greatly facilitates users and broadens the application field of wireless sensor networks.

\section{Overview of The Basic Theory of Socket Communication}

\subsection{TCP/IP Protocol in WinCE}

$\mathrm{TCP} / \mathrm{IP}$ protocol is a complete set of network protocols on the physical network. It is a network architecture of 4 layers: application layer, transport layer, Internet layer and data link layer. TCP/IP protocol stack is the core of WinCE network, and network communication of embedded devices directly or indirectly uses WinSock port socket to access to the TCP/IP protocol network stack.

\subsection{Overview of The Principles And Mechanisms of Socket Communication}

In the early 1990s, Microsoft and third party vendors developed a common standard: the Windows Sockets specification, namely Winsock. Windows Sockets network programming interface specification is a set of open, multiple protocols, which arw based on Windows operating system, a standard Internet protocol (IPS, general TCP/IP) API, which is used to shield the implementation details of the network.In the Winsock, a socket object is the endpoint of a communication and can send and receive data packets over the network through sockets. Under TCP/IP, the socket is defined by the IP address and port number. The IP address includes the $4 \mathrm{~B}$, which is used to identify the servers on the Internet[2]. The port number identifies a particular function or service provided by the server and is associated with an application. The application can choose its own port number between 1024 and 65535 .

\section{Design of System Architecture}

The design of system architecture is shown in Figure 1 as below. The wireless sensor network with the ZigBee wireless communication technology is based on IEEE 802.15.4 standard and coordinates thousands of tiny sensors to achieve efficient communication. The 
system uses the base station with the S3C2440 processor as a development module of ARM9 controller while ARM9 is equipped with the WinCE6.0 real-time operating system. In order to achieve the temporary storage of data in wireless sensor networks, the Microsoft SQL Server 2005 Mobile Edition database is embedded in the system. By maintaining the technical advantages of the traditional remote control system, combined with the actual functional requirements, the network programming based on Sockets communication technology is adopted to realize the remote control function[3].

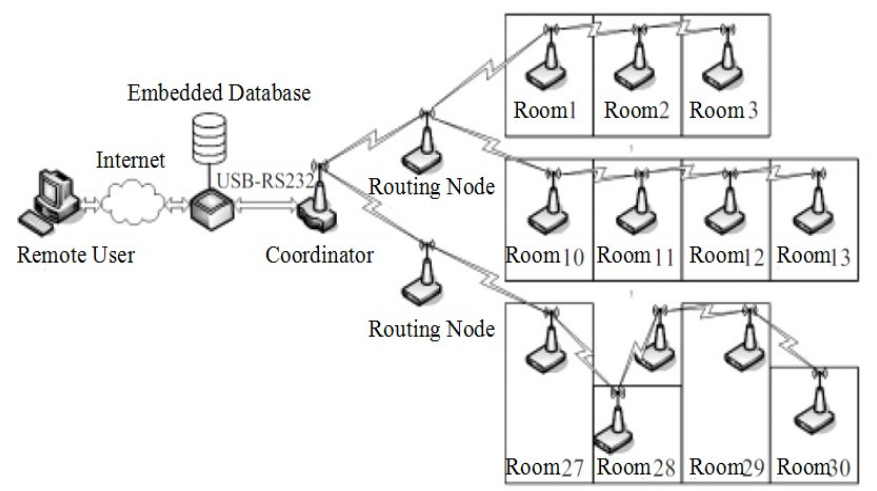

Figure 1:System Architecture of ZigBee Network

\section{Design of System Hardware}

The whole hardware system configures the server JN5148 and PIC16f723, among which, JN5148 is used to realize the wireless data transceiver based on ZigBee network protocol. It is the hardware base of communication. PIC16f723 is used to realize the power management of hardware system[3]. The third generation ZigBee chipdeveloped by JENNIC company with the characteristics of low power consumption and strong processing ability is selected.According to the working requirements of the node, the power management circuit can set the working cycle of the node. Besides, it can realize the timing control of the load switch through the single chip microcomputer programming and achieve the effect of energy saving.The JN5148 in UART0 is transformed into USB type T serial port to realize the communication between node and base station and improve the versatility of the node.

\section{Design of System Software}

\subsection{Set Up The Development Environment}

Let's install the following softwares on the PC:Microsoft Visual Studio 2005; Microsoft SQL Server 2005;Microsoft ActiveSync;CE based Windows SDK:Microsoft SQL Server 2005 Mobile Edition database.

\subsection{Programming Design ofSockets Network}

In order to realize Sockets communication between ARM base stations and remote clients, it is necessary to design ARM base station server and remote PC clients respectively. The communication process of this system is shown in figure 2 . 


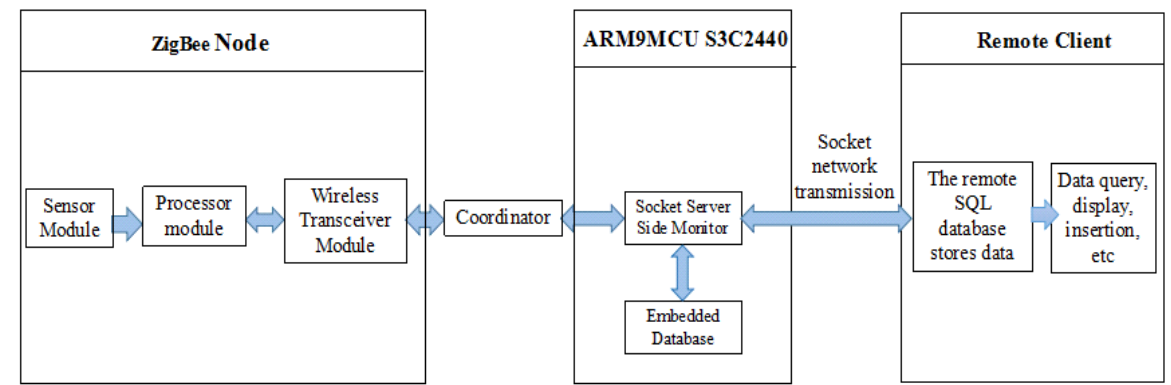

Figure 2: Flow Chart of System Communication

The server interface is divided into four modules, which are operational management, online management, data transmission and data monitoring. Operational management module receives the server information. Real-time monitoring of the port is used to establish a connection with the remote computer. Online management module is used to realize the online communication between the server and client, sending information to each other. Data transmission module is the temperature and humidity data stored in the embedded database SQLCE to the already established connection client. To achieve real-time monitoring,we shall have data monitoring module in the operation state of the wireless sensor network[4].

The client main implementation function has 3 aspects:

1) send connection request based on the server's IP and establish a connection with the server;

2) send information to server online and receive information from the server in real time;

3) monitor all network data streams of the machine, analyze its source address and size, and generate a log.

\subsubsection{The Principle of System Socket Communication}

Combined with the actual application background of the system and the characteristics of TCP and UDP, this system uses TCP programming. Socket communication principle is mainly reflected in the server and the client can establish the connection, send and receive data from each other in the process[5]. The specific program flow is shown in figure 3.

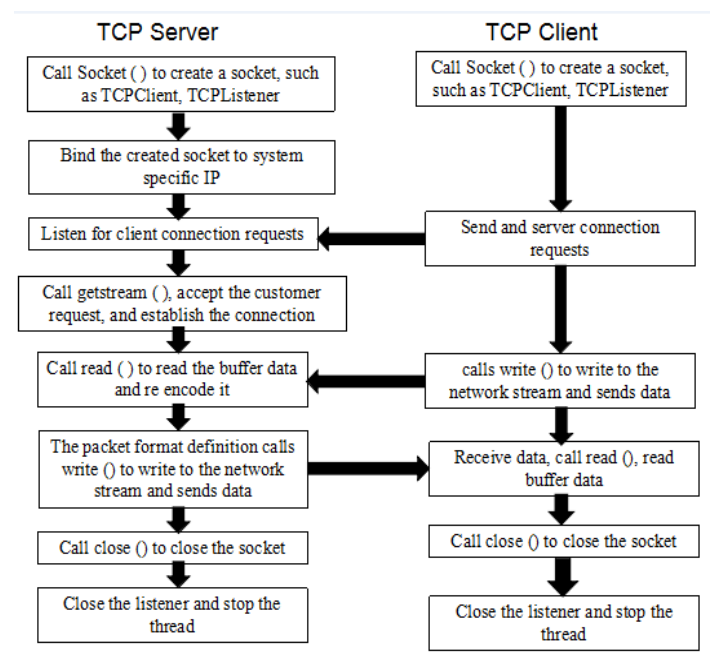

Figure 3: TCP Server /Client Programming Process 


\section{Implementation of The System}

The experimental system uses a wireless sensor network system developed by our research team, which has 30 nodes. Figure 4 shows the hardware diagram of the 5 nodes in the system. The rest nodes have the same hardware design, and different functions are realized by downloading different programs. In the diagram, the coordinating node is responsible for connecting the embedded base stations. The other are the routing nodes and the underlying nodes[6].

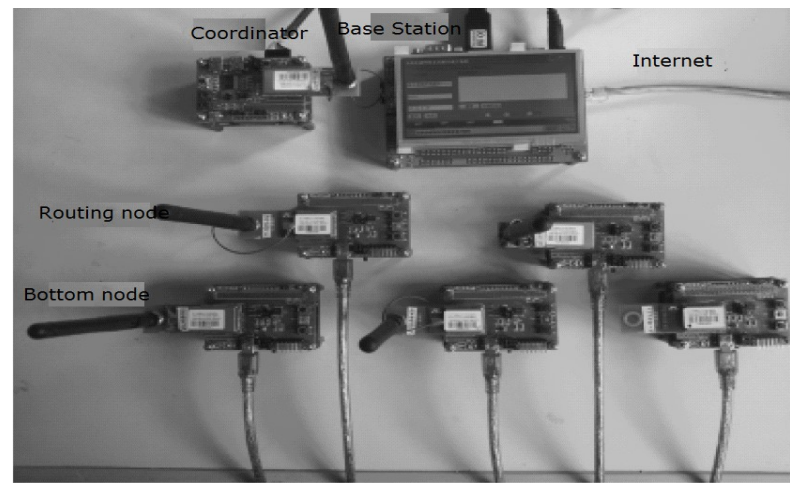

Figure 4: WSNs System Diagram

\subsection{Realization of Data Transmission of Temperature And Humidity}

\subsubsection{Server Function Implementation}

As shown in Figure 5, the WinCE base station serves main interfaces. Operational management module monitors the port information for real-time. The port 6001 (from 1024 to 65535 within the allowable range of custom) is waiting for establishing the connection with the remote clients[7].

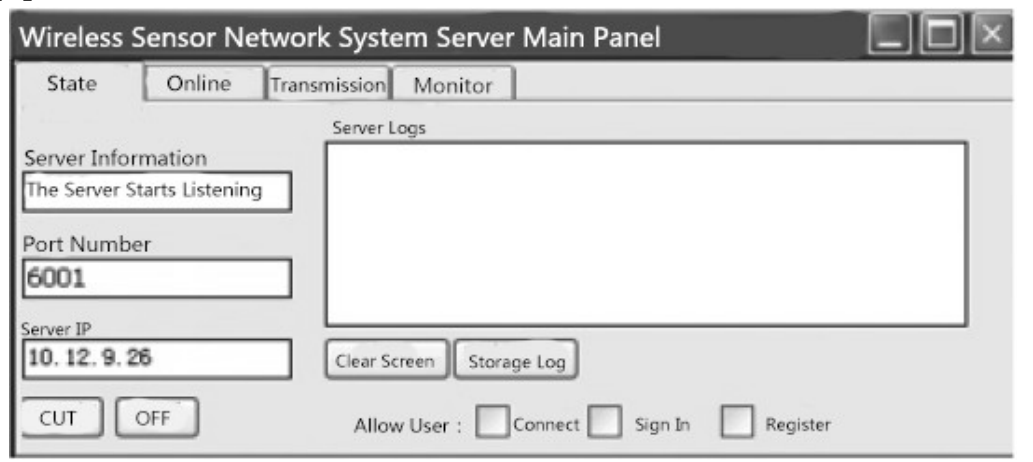

Figure 5: Monitor Port, Waiting for Connection

By running the server data transfer module, the data is extracted from the embedded database and sent to the remote clients. The interface in Figure 6 shows the data in the extracted base station SQLCE. Click the button to send the database data, and the COM port console listens to the data and sends the data to the remote users. When data is transmitted, the packet format can be defined as:

(a) number|time|node|temperature|humidity|.

For example, when sending 426th records in SQLCE, the packet format is @426 |2016-0601 01:08:44| $\mid$ node $=10 \mid$ temp $=28 \mid$ humid $=51 \mid$. 
Among them, @ represents a record, | represents the column of a record in the SOLCE. When the client receives the data packet, it can unpack data packets according to the format definition,so as to extract the effective data.

\begin{tabular}{|c|c|c|c|c|}
\hline \multicolumn{4}{|c|}{ Wireless Sensor Network System Server Main Panel } & $-\square[x$ \\
\hline State & Online Transmission & \multicolumn{2}{|c|}{ Monitor } & \\
\hline Serial Number & Time & Node & Temperature & Humidity $\wedge$ \\
\hline 3 & $2016-06-01$ 01:06:28 & node $=22$ & temp $=28$ & humid=! \\
\hline 4 & $2016-06-0101: 06: 31$ & node $=10$ & temp $=28$ & humid=! \\
\hline 6 & $2016-06-0101: 08: 39$ & node $=10$ & temp $=28$ & humid=! \\
\hline 7 & $2016-06-0101: 08: 42$ & node $=22$ & temp $=28$ & humid=! \\
\hline 8 & 2016-06-01 01:08:44 & node $=10$ & temp $=28$ & humid=? \\
\hline 9 & 2016-06-01 01:08:47 & node $=22$ & temo $=28$ & humid $=$ th \\
\hline$\leqslant$ & & & & $>1: 3$ \\
\hline
\end{tabular}

Figure 6: Extract Data in Embedded Database SQLCE of Base Station(BS)

\subsubsection{Implementation of System ClientFunction}

When the remote client successfully establishes a connection with the server, the data sent from the base station is stored in the SQL database. By accessing to the local SQL database, the client can query the data sent from the base station server[8].

\subsection{Implementation of Online Management Function}

When the client sends a request to the server, the server receives the instruction[9]. It will send the relevant data stored in the base station to the user according to the request, so as to realize the online data management, as shown in Figure $7 \mathrm{a}, \mathrm{b}$.

\begin{tabular}{|c|c|c|c|c|}
\hline \multicolumn{5}{|c|}{ Wireless Sensor Network System Server Main Panel } \\
\hline State & Online Transmission] & Monitor & & \\
\hline \multicolumn{3}{|l|}{ Send Data } & \multicolumn{2}{|c|}{ Receive Data } \\
\hline \multicolumn{2}{|c|}{$\begin{array}{l}2016-06-0101: 01: 20 \text { node }=10 \\
\text { temp }=27 \text { humid }=53 ; 2016-06-01 \\
05: 23: 29 \text { node }=10 \text { temp }=26\end{array}$} & & \multicolumn{2}{|c|}{$\begin{array}{l}\text { Operator: } \\
\text { Send two sets of data } 10 \text { 2016-06-01 } \\
01: 00-02: 00 \text { Temperature and humidity data }\end{array}$} \\
\hline \multirow[t]{2}{*}{ Send Out } & Close Down & & & \\
\hline & & & Select File & Send File \\
\hline
\end{tabular}

Figure 7a: The Server Sends and Receives Data Online

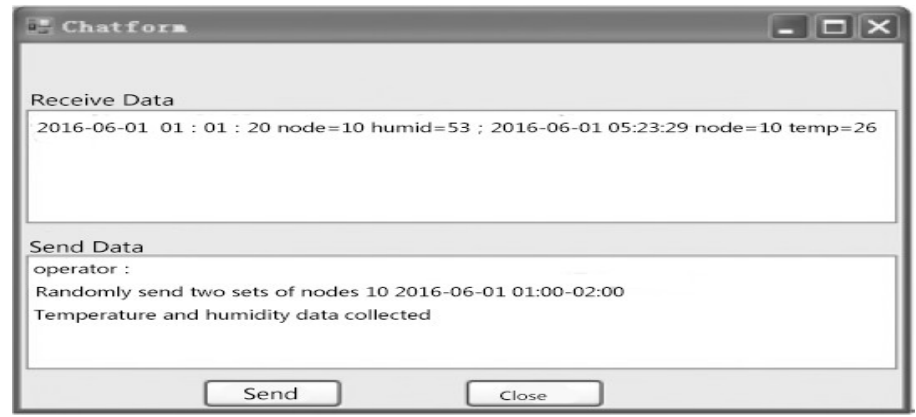

Figure 7b: The Client Sends and Receives Data Online

\subsection{Implementation of Network Monitor}

The network monitor can monitor the network flow of the client in real time, analyzing each data packet and thus obtaining the protocol type,the source port,the target address and the length of the packet for each data packet[10]. 


\section{Conclusion}

In this paper, the research group took the monitoring of a building's temperature and humidity data as an example. With the use of Windows network programming technology, Visual Studio 2005 development environment and C\# programming language, together with the combination of Sockets socket and the embedded SQLCE database development technology, the research group successfully developed a Socket communication system based on wireless sensor network technology. The experiment shows that the socket communication system can make the remote computer realize the real-time display, storage and query of the data of the wireless sensor network. This system not only can be applied to handle some of the traditional communication needs, such as building energy monitoring, industrial process monitoring and fault monitoring, but also be adapt to the new environment of network application, such as network education, video data transmission and some other fields.

\section{References}

[1]Suppressing failure cascades in interconnected networks: Considering capacity allocation pattern and load redistribution[J]. Modern Physics Letters B, 2016, 30(05): 1650049.

[2]Jun Sheng Xiao, Yu Gang Dai,Tao Xu. The Model Used to Pretect Wetlands Based on Zigbee Technology[J]. Applied Mechanics and Materials,2013,2658(397)

[3]Zheng Hua Zhang,Jun Gu,Yi Ming Zhao,Ping Liu. The Design of Heterogeneous Network Integration Gateway System Based on Multi-Threaded[J]. Applied Mechanics and Materials,2014,3560(667)

[4]Peng Ju Zhang,Gai Zhi Guo,Zong Zuo Yu. Application of Wireless Sensor Network in Embedded Smart Home System[J]. Applied Mechanics and Materials,2015,3785(738)

[5]César Ortega-Corral,Luis E. Palafox,J. Antonio García-Macías,Jaime Sánchez-García,Leocundo Aguilar,Tai-hoon Kim. End-to-End Message Exchange in a Deployable Marine Environment Hierarchical Wireless Sensor Network[J]. International Journal of Distributed Sensor Networks,2014

[6] Cascading failure analysis and restoration strategy in an interdependent network [J]. Journal of Physics A: Mathematical and Theoretical, 2016, 49(19): 195101.

[7]Hong Yaoming, Lin Hsueh-Chun, Kan Yao-Chiang. Using wireless sensor network on real-time remote monitoring of the load cell for landslide $[\mathrm{J}]$. Sensor Letters, 2011, 9( 5) : 19111915.

[8]Epidemic spreading model of complex dynamical network [J]. International Journal of Systems Science, 2015, 1-8.

[9]Safar Maytham, Al-Hamadi Hasan, Ebrahimi Dariush.PECA: Power efficient clustering algorithm for wireless sensor networks[J].International Journal of Information Technology and Web Engineering,2011,6(1) : 49-58

[10]S. Elango,N. Mathivanan,Pankaj Gupta. RSSI Based Indoor Position Monitoring Using WSN in a Home Automation Application[J]. Acta Electrotechnica et Informatica,2011,11(4). 